\title{
AN OPTIMIZATION METHOD TO FIND THE INITIAL CATENARY CONFIGURATION BY USING A GRADIENT-BASED ALGORITHM
}

\author{
Kee Seung $\mathrm{Oh}^{1}$, Rosalie Morin ${ }^{1}$, Ben Shiff ${ }^{1}$, Stephen William Knox Roper ${ }^{1}$, Il Yong Kim ${ }^{1 *}$ \\ ${ }^{1}$ Department of Mechanical and Materials Engineering, Queen's University, Kingston, Canada \\ *kimiy@queensu.ca
}

\begin{abstract}
In this study, an optimization method is proposed to obtain an initial configuration of the catenary. To this end, a gradient-based algorithm is employed, and the sensitivity analysis is performed by introducing an alternative finite difference method (FDM). Unlike the original FDM, a proposed method can dramatically reduce the computation cost due to its simplified format. The form-finding problem is formulated as the unconstrained optimization problem with an objective function defined by half mean squared error. In the optimization process, static analysis for the catenary constructed by the 2-node beam elements is performed at each iteration calculation using commercial software. A welldefined unconstrained optimization problem is solved successfully, and the validity of the suggested optimization method is supported by the numerical results obtained for specific design conditions.
\end{abstract}

Keywords- pantograph-catenary system; static analysis; optimization; finite different method

\section{INTRODUCTION}

The overhead line system for a high-speed modern train should be operated under the stable current-collection quality which can be judged by the contact loss between the catenary and pantograph. Numerical simulations are required to verify performance primarily because real-world overhead line tests are inefficient, expensive and sometimes dangerous. The simulation for the high-speed train should capture exactly the wave propagation phenomena in the catenary system because it dominates the physical behaviors between the pantograph and catenary [1]. In order to simulate the pantograph-catenary system accurately, two numerical methods have been intensively investigated so far: the absolute nodal coordinate formulation (ANCF) based finite element method (FEM) [2-9] and the standard FEM [10-16]. In a nutshell, the standard FEM considers six degrees of freedom (DOF) for the 2-node beam element, but the ANCF based FEM solves for 12 DOF for the same beam element because it additionally considers the absolute nodal coordinates to calculate the large deformation phenomenon effectively.

There is another important issue in the simulation to obtain the stable current-collection quality apart from the formulation

\footnotetext{
NSERC, SNC-Lavalin
}

selection of the numerical methods: initial catenary formfinding problem considering pre-sag and distortion. Since the catenary configuration is exerted by the external forces such as gravitational and tensile forces, the pre-sag and distortion of the catenary are inevitable if one does not perform optimization for finding proper initial catenary configuration. Accordingly, numerous methods have been reported to overcome this issue so far. There were useful form-finding methods that considered the constraints introduced during the assembly of the catenary, pantograph and the other parts [4,7], or found the static form of the catenary configuration by using geometry variation method [11,12]. Also, Zhou et al. [13] performed the optimization process by introducing the negative sag method, Massat et al. identified the dropper length by minimizing the dropper tension error in [14], and Yang et al. [8] facilitated the catenary formfinding by controlling tension with the piecewise equations. Several types of research with respect to minimizing distance have been reported. Ambrósio et al. [15] performed minimizing the distance between the static deformed geometry of the contact wire and its specified position and Collina et al. minimized a residual function constructed by the weighted differences between the target and the design values of the tensile load in the wires and the lateral position of the steady arms in [16]. Besides, Gregori et al. [9] minimized the interaction force between the catenary wire and pantograph head by using a genetic algorithm. However, there was a lack of optimization formulation descriptions in the researches mentioned previously to the catenary form-finding problems.

Even though a tailored analytical solution was proposed in [17], it was only valid for the specific geometry and parameters. Therefore, an advanced optimization scheme should be developed to obtain the proper initial configuration for the various catenary types. In this study, a new optimization method is proposed to solve the form-finding problem by using a gradient-based algorithm with an alternative finite difference method (FDM) for the sensitivity analysis. This paper is organized as follows: Section II describes the static analysis method with the governing equation and boundary conditions. Section III describes the optimization formulation for the catenary initial form-finding problem. In Section IV, the optimal results are shown with the optimization history and tabulated data. 


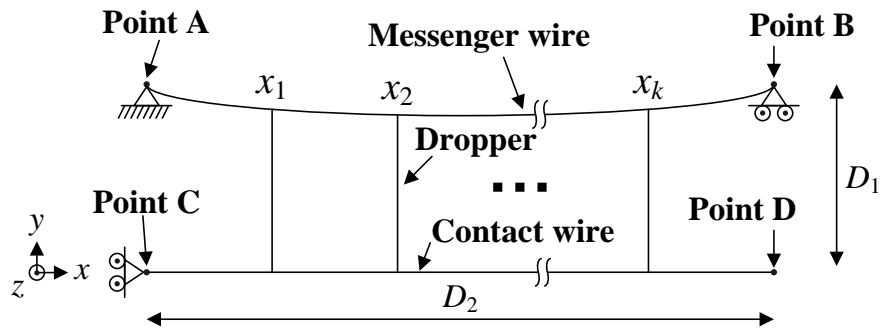

Figure 1. Two-dimensional catenary model

\section{CATNEARY STATIC ANALYSIS}

In this section, a numerical analysis method is described for obtaining the static catenary configuration before proposing a new method to solve the form-finding problem.

\section{A. Simulation Model}

A single-span catenary configuration is selected for the analysis and optimization model. Its geometry is shown in Fig. 1 , and there is an assumption that the model only behaves on the $x-y$ plane considering only in-plane degrees of freedom. The height $\left(D_{1}\right)$ and length $\left(D_{2}\right)$ of the span are $1.2[\mathrm{~m}]$ and 60 $[\mathrm{m}]$, respectively. The number of droppers and their heights and locations will be shown in the following section.

\section{B. Govering Equation and Boundary Conditions}

The displacements of for the contact $\left(u_{\mathrm{cw}}\right)$ and messenger wires $\left(u_{\mathrm{mw}}\right)$ denoted by the subscripts ' $\mathrm{cw}$ ' and 'mw', respectively, are governed by Euler-Bernoulli beam theory as follows.

$$
\begin{gathered}
E I_{\mathrm{cw}} \frac{\partial^{4} u_{\mathrm{cw}}}{\partial q^{4}}-T_{\mathrm{cw}} \frac{\partial^{2} u_{\mathrm{cw}}}{\partial q^{2}}+\sum_{k=1}^{K} f_{k}^{\mathrm{dr}} \delta\left(q-x_{k}\right)=0, \\
E I_{\mathrm{mw}} \frac{\partial^{4} u_{\mathrm{mw}}}{\partial q^{4}}-T_{\mathrm{mw}} \frac{\partial^{2} u_{\mathrm{mw}}}{\partial q^{2}}-\sum_{k=1}^{K} f_{k}^{\mathrm{dr}} \delta\left(q-x_{k}\right)=0,
\end{gathered}
$$

where $q$ represents the local coordinate in the longitudinal direction of the beam element. $E I$ and $T$ stand for the bending stiffness and tensile force of wires, respectively. $f_{k}^{\mathrm{dr}}$ and $x_{k}$ represent an external force by a dropper and the location of $k$ th dropper, respectively. For the messenger wire in Fig. 1, Point $\mathrm{A}$ is constrained against displacements in $x$ and $y$ directions, and Point $\mathrm{B}$ is constrained against displacement $y$, and a tensile force $T_{m}$ is applied on Point B as well. For the contact wire, Point $\mathrm{C}$ is constrained against displacement in $x$ direction and rotation about $z$. Point $\mathrm{D}$ is constrained against rotations $z$, and exerted a tensile force $T_{c}$. Further, all nodes are globally constrained against displacements in $z$ and rotations about $x$ and $y$ and a gravitational force is applied to all nodes in the $-y$ direction. Based on the governing equation and boundary conditions, the standard FEM formulation can be constructed as in (3).

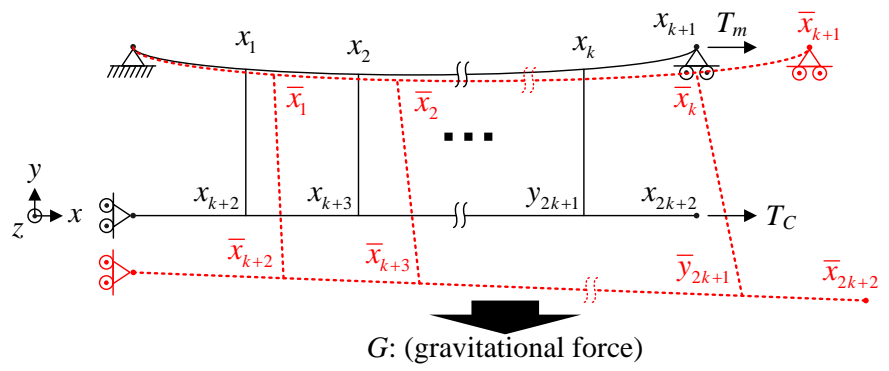

(a)

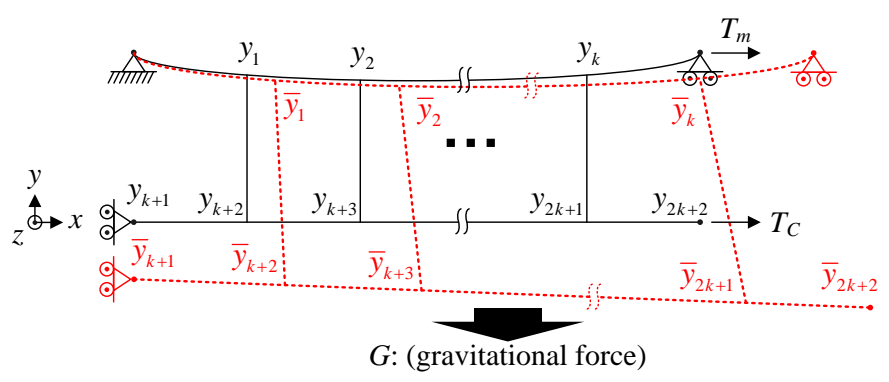

(b)

Figure 2. Keypoints for undeformed and deformed configurations represented by black-solid and red-dashed lines. (a) Keypoints for $x$ direction. (b) Keypoints for $y$ direction.

$$
\mathbf{K u}=\mathbf{f},
$$

where $\mathbf{K}, \mathbf{f}$ and $\mathbf{u}$ denote a global mass matrix, the vector of external forces and the vector of nodal displacement, respectively.

\section{Static Analysis Implementation}

To obtain the static solution in (3), ANSYS APDL script [18] is used with the 2-node Euler-Bernoulli beam element (BEAM188) for the messenger and contact wires modeling. Also, the tensile forces of wires due to droppers, $f_{k}^{\text {dr }}$ in (1) and (2), can be modeled by using the non-linear spring element (COMBIN39). The deformed and undeformed coordinates for keypoints as shown in Fig. 2 can be used to construct the vectors outlined in (4) and (5). A Python scrip is used to integrate the ANSYS APDL script in (6) with the form-finding optimization problem.

$$
\begin{gathered}
\chi=\left\{x_{1}, \ldots, x_{m}, \ldots, x_{M}, y_{1}, \ldots, y_{n}, \ldots, y_{N}\right\}^{T}, \\
\bar{\chi}=\left\{\bar{x}_{1}, \ldots, \bar{x}_{m}, \ldots, \bar{x}_{M}, \bar{y}_{1}, \ldots, \bar{y}_{n}, \ldots, \bar{y}_{N}\right\}^{T}, \\
\bar{\chi}=f_{\text {Static }}^{\text {APDL }}(\chi),
\end{gathered}
$$

where $\chi$ and $\bar{\chi}$ represent the undeformed and deformed coordinates vectors, respectively, and the number of the coordinates are $M=N=2 K+2$. 


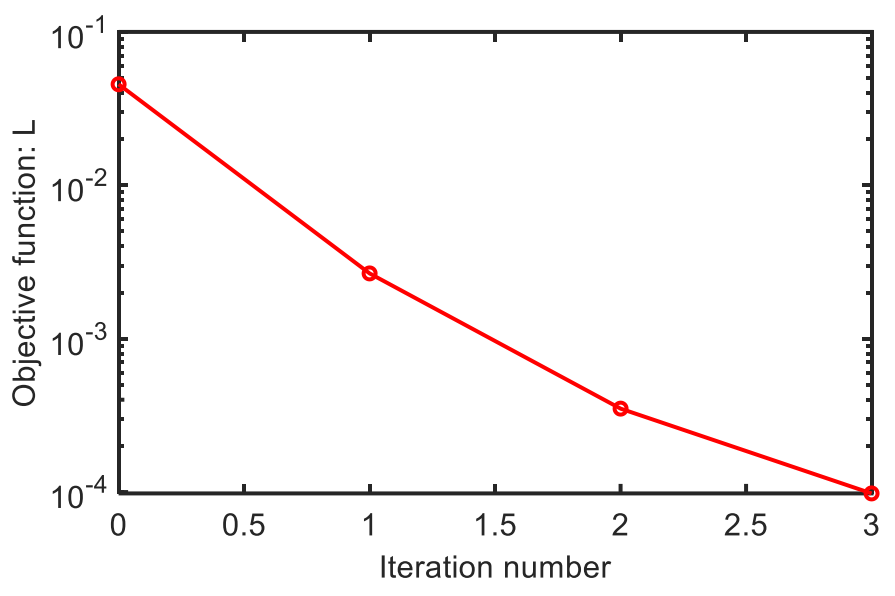

Figure 3. Optimization history

\section{CATENARY OPTIMIZATION FormULATION}

\section{A. Set the Target Values for Keypoints}

In this study, a single span catenary configuration with 9 droppers $(K=9)$ is considered, so the number of the keypoints for $x$ and $y$ coordinates in (4) and (5) are $M=20$ and $N=20$, respectively. The $x$ direction coordinates for the target keypoints are defined by EN50318, and the $y$ direction coordinates are obtained considering the momentum equilibrium as described in [5,6]. Also, the zero pre-sag is only considered in this study, so the $y$ direction target values for the contact wires are all zeros. The target values are listed in TABLE. I, and the values are compiled in $\chi^{\text {target }}$ as follows with the total number of the target values, $S=40$.

$$
\begin{aligned}
\chi^{\mathrm{target}} & =\left\{\chi_{1}^{\mathrm{target}}, \ldots, \chi_{s}^{\mathrm{target}}, \ldots, \chi_{S}^{\mathrm{target}}\right\}^{T} \\
& =\left\{x_{1}^{\mathrm{target}}, \ldots, x_{M}^{\mathrm{target}}, y_{1}^{\mathrm{target}}, \ldots, y_{N}^{\mathrm{target}}\right\}^{T} .
\end{aligned}
$$

\section{B. Objective Function and Sensitivy Analysis}

The form-finding problem can be solved by optimizing the unconstrained optimization problem with the design variable vector $\chi$. To this end, an objective function is defined in (8) and (9) which is half mean squared error.

$$
\begin{gathered}
\min _{\chi} L, \\
L(\boldsymbol{\chi})=\frac{1}{2} \mathbf{1} \cdot\left[\left(\chi^{\mathrm{target}}-\bar{\chi}\right) \circ\left(\chi^{\mathrm{target}}-\bar{\chi}\right)\right] \\
=\frac{1}{2} \sum_{s=1}^{S}\left(\chi_{s}^{\mathrm{target}}-\bar{\chi}_{s}\right)^{2},
\end{gathered}
$$

where ' ' and ' $\circ$ ' represent the dot and Hadamard product operations, respectively.

In order to minimize the objective function $L$ using the gradient-based algorithms, sensitivity analysis should be performed preliminary: $\partial L / \partial \chi$. Unfortunately, since the objective function is calculated based on a black-box-like
TABLE I. TARGET VALUES FOR FORM-FINDING OPTIMIZATION

\begin{tabular}{|c|c|c|c|}
\hline Symbols & Values [m] & Symbols & Values [m] \\
\hline$x_{1}^{\text {target }}$ & 5.0 & $y_{1}^{\text {target }}$ & 0.985636172 \\
\hline$x_{2}^{\text {target }}$ & 10.5 & $y_{2}^{\text {target }}$ & 0.804060736 \\
\hline$x_{3}^{\text {target }}$ & 17.0 & $y_{3}^{\text {target }}$ & 0.647338322 \\
\hline$x_{4}^{\text {target }}$ & 23.5 & $y_{4}^{\text {target }}$ & 0.553304873 \\
\hline$x_{5}^{\text {target }}$ & 30.0 & $y_{5}^{\text {target }}$ & 0.521960391 \\
\hline$x_{6}^{\text {target }}$ & 36.5 & $y_{6}^{\text {target }}$ & 0.553304873 \\
\hline$x_{7}^{\text {target }}$ & 43.0 & $y_{7}^{\text {target }}$ & 0.647338322 \\
\hline$x_{8}^{\text {target }}$ & 49.5 & $y_{8}^{\text {target }}$ & 0.804060736 \\
\hline$x_{9}^{\text {target }}$ & 55.0 & $y_{9}^{\text {target }}$ & 0.985636172 \\
\hline$x_{10}^{\text {target }}$ & 60.0 & $y_{10}^{\text {target }}$ & 0 \\
\hline$x_{11}^{\text {target }}$ & $x_{1}^{\text {target }}$ & $y_{11}^{\text {target }}$ & 0 \\
\hline$x_{12}^{\text {target }}$ & $x_{2}^{\text {target }}$ & $y_{12}^{\text {target }}$ & 0 \\
\hline$x_{13}^{\text {target }}$ & $x_{3}^{\text {target }}$ & $y_{13}^{\text {target }}$ & 0 \\
\hline$x_{14}^{\text {target }}$ & $x_{4}^{\text {target }}$ & $y_{14}^{\text {target }}$ & 0 \\
\hline$x_{15}^{\text {target }}$ & $x_{5}^{\text {target }}$ & $y_{15}^{\text {target }}$ & 0 \\
\hline$x_{16}^{\text {target }}$ & $x_{6}^{\text {target }}$ & $y_{16}^{\text {target }}$ & $y_{20}^{\text {target }}$ \\
\hline$x_{17}^{\text {target }}$ & $x_{7}^{\text {target }}$ & $y_{17}^{\text {target }}$ & 0 \\
\hline$x_{18}^{\text {target }}$ & $x_{18}^{\text {target }}$ & $y_{19}^{\text {target }}$ & 0 \\
\hline$x_{19}^{\text {target }}$ & $x_{20}^{\text {target }}$ & & 0 \\
\hline
\end{tabular}

function as shown in (6), analytical sensitivity analysis cannot be performed. Finite difference method (FDM) can be use instead, however, this significantly increases computational costs.

$$
\frac{\partial L}{\partial \chi}=\left\{\begin{array}{c}
\left(\chi_{1}^{\text {target }}-\bar{\chi}_{1}\right) \frac{\bar{\chi}_{1}^{\prime}-\bar{\chi}_{1}}{\Delta \chi_{1}} \\
\vdots \\
\left(\chi_{s}^{\text {target }}-\bar{\chi}_{s}\right) \frac{\bar{\chi}_{s}^{\prime}-\bar{\chi}_{s}}{\Delta \chi_{s}} \\
\vdots \\
\left(\chi_{s}^{\text {target }}-\bar{\chi}_{s}\right) \frac{\bar{\chi}_{s}^{\prime}-\bar{\chi}_{s}}{\Delta \chi_{s}}
\end{array}\right\},
$$


where $\Delta$ stands for a small change of the corresponding variable, and $\bar{\chi}_{s}^{\prime}$ denotes the deformed coordinates considering $\Delta$. As shown in (10), the original FDM requires $S+1$ separate finite element analyses which increases computation cost. Therefore, an alternative FDM is proposed in (11) with an assumption that $\bar{\chi}_{s}^{\prime}-\bar{\chi}_{s}$ is almost identical to $\Delta \chi_{s}$.

$$
\frac{\partial L}{\partial \chi}=\chi^{\text {target }}-\bar{\chi}
$$

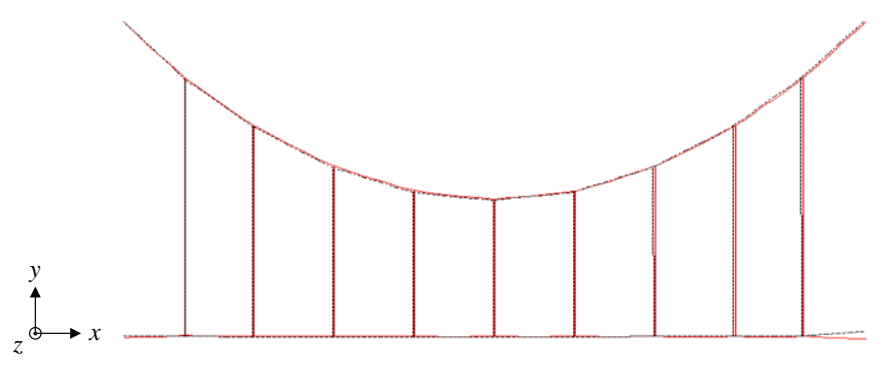

Figure 4. The optimal catenary (black-dashed line) and deformed catenary based on the optimal keypoints (red-solid line) congifurations.

TABLE II. DIFFERENCE BETWEEN THE TARGET AND DEFORMED KEYPOINTS

\begin{tabular}{|c|c|c|c|}
\hline $\begin{array}{l}\text { Design variable } \\
\text { index }(S)\end{array}$ & $\chi^{\text {target }}-\bar{\chi}^{\text {opt }}[\mathrm{m}]$ & $\begin{array}{l}\text { Design variable } \\
\text { index }(S)\end{array}$ & $\chi^{\text {target }}-\bar{\chi}^{\text {opt }}[\mathrm{m}]$ \\
\hline 1 & -0.000192 & 21 & -0.000572 \\
\hline 2 & -0.000104 & 22 & -0.002698 \\
\hline 3 & -0.000084 & 23 & -0.002821 \\
\hline 4 & -0.000064 & 24 & -0.002786 \\
\hline 5 & -0.000043 & 25 & -0.002629 \\
\hline 6 & -0.000241 & 26 & -0.002355 \\
\hline 7 & -0.000011 & 27 & -0.001920 \\
\hline 8 & -0.000020 & 28 & -0.001632 \\
\hline 9 & -0.000033 & 29 & -0.001527 \\
\hline 10 & 0.000011 & 30 & 0.004299 \\
\hline 11 & 0.000016 & 31 & -0.003862 \\
\hline 12 & 0.000034 & 32 & -0.002503 \\
\hline 13 & 0.000050 & 33 & -0.002572 \\
\hline 14 & 0.000066 & 34 & -0.002504 \\
\hline 15 & 0.000082 & 35 & -0.002277 \\
\hline 16 & 0.000100 & 36 & -0.001884 \\
\hline 17 & 0.000113 & 37 & -0.002981 \\
\hline 18 & 0.000126 & 38 & -0.000491 \\
\hline 19 & 0.000142 & 39 & -0.001957 \\
\hline
\end{tabular}

\begin{tabular}{|c|c|c|c|}
\hline $\begin{array}{c}\text { Design variable } \\
\text { index }(\boldsymbol{S})\end{array}$ & $\chi^{\text {target }}-\bar{\chi}^{\text {opt }}[\mathrm{m}]$ & $\begin{array}{c}\text { Design variable } \\
\text { index }(\boldsymbol{S})\end{array}$ & $\chi^{\text {target }}-\bar{\chi}^{\text {opt }}[\mathrm{m}]$ \\
\hline 20 & 0.000075 & 40 & 0.008825 \\
\hline
\end{tabular}

C. Optimization Algorithm

The Broyden-Fletcher-Goldfarb-Shanno (BFGS) algorithm is selected as an optimizer for the unconstrained optimization problem formulated in the previous section. There are many options in the algorithm selection, but the optimizers such as Newton's method which requires the Hessian matrix are not suitable in the optimization formulation as in (8) because of the high computational cost in FDM. Therefore, the BFGS method having high accuracy for the search direction and step length based only on the gradient vector is employed as the optimization algorithm in our problem. To implement this algorithm in the Python environment, SciPy is introduced, [19] which is a free and open-source Python library.

\section{OPTIMIZATION RESULTS AND DISCUSSIONS}

\section{A. Optimization History}

The form-finding problem is successfully solved by minimizing the objective function in (8). The optimization history is displayed in Fig. 3, and shows the efficient convergence behavior. These results conclude that the simplified FDM in (11) works for the catenary configuration optimization problem.

\section{B. Optimization Result Validation}

The mathematical relationship between the optimal ( $\left.\chi^{\text {opt }}\right)$ and deformed $\left(\bar{\chi}^{\text {opt }}\right)$ keypoints in (12) as follows.

$$
\bar{\chi}^{\mathrm{opt}}=f_{\text {Static }}^{\mathrm{APDL}}\left(\chi^{\mathrm{opt}}\right) \text {. }
$$

The optimal and deformed catenary configurations are displayed in Fig. 4 represented by black-dashed line based on $\chi^{\text {opt }}$ and red-solid line based on $\bar{\chi}^{\text {opt }}$, respectively. The difference between the target values and deformed values based on the optimal coordinates should be close to $\mathbf{0}$ as represented in (13), and TABLE. II lists these values.

$$
\chi^{\text {target }}-\bar{\chi}^{\text {opt }} \cong \mathbf{0} \text {. }
$$

Consequently, the optimization results in TABLE.II show a capability of the proposed method to obtain a proper initial form of catenary. This method can be used as the preliminary stage of the transient simulation for the pantograph-catenary system.

\section{ACKNOWLEDGMENT}

This research was funded by the Natural Sciences and Engineering Research Council of Canada (NSERC) and Industry Partner SNC-Lavalin. Technical advice and guidance were gratefully received from Samantha Lee, Anesh Ramkhelawon, Dion Church, Hassan Nasri, Manjodh Banwait and Richard Catlow. Their expertise was greatly appreciated. 


\section{REFERENCES}

[1] EN 50318:2002, Railway applications - current collection systems current collection systems validation of simulation of the dynamic interaction between pantograph and overhead contact line. European Committee for Electrotechnical Standardization, 2002.

[2] J. H. Seo, H. Sugiyama, and A. A. Shabana, "Three-Dimensional Large Deformation Analysis of the Multibody Pantograph/Catenary Systems," Nonlinear Dyn., vol. 42, pp. 199-215, February 2005.

[3] J. H. Seo, S. W. Kim, I. H. Jung, T. W. Park, J. Y. Mok, Y. G. Kim, and J. B. Chai, "Dynamic analysis of a pantograph catenary system using absolute nodal coordinates," Veh. Syst. Dyn., vol. 44, pp. 615-630, August 2006.

[4] M. Tur, L. Baeza, F. J. Fuenmayor, and E. García, "PACDIN statement of methods," Veh. Syst. Dyn., vol. 53, pp. 402-411, March 2015.

[5] S. P. Jung , Y. G. Kim , J. S. Paik, and T. W. Park, "Estimation of dynamic contact force between a pantograph and catenary using the finite element method," J. Comput. Nonlinear Dynam., vol. 7, pp. 041006, June 2012.

[6] J. H. Lee and T. W. Park, "Development of a three-dimensional catenary model using cable elements based on absolute nodal coordinate formulation," J. Mech, Sci. Technol., vol. 26, pp. 3933-3941, December 2012.

[7] M. Tur, E. García, L. Baeza, and F. J. Fuenmayor, "A 3D absolute nodal coordinate finite element model to compute the initial configuration of a railway catenary," Eng. Struct., vol. 71, pp. 234-243, July 2014.

[8] C. J. Yang, W. H. Zhang, J. Zhang, G. M. Mei, N. Zhou, and G. X. Ren, "Static form-finding analysis of a railway catenary using a dynamic equilibrium method based on flexible multibody system formulation with absolute nodal coordinates and controls," Multibody Syst. Dyn., vol. 39, pp. 221-247, March 2017.

[9] S. Gregori, M. Tur, E. Nadal, and F. J. Fuenmayor, "An approach to geometric optimisation of railway catenaries," Veh. Syst. Dyn., vol. 56, pp. 1162-1186, August 2018.

[10] Y. H. Cho, "Numerical simulation of the dynamic responses of railway overhead contact lines to a moving pantograph, considering a nonlinear dropper," J. Sound Vib., vol. 315, pp. 433-454, August 2008.

[11] Y. H. Cho, K. Lee, Y. Park, B. Kang, and K. Kim, "Influence of contact wire pre-sag on the dynamics of pantograph-railway catenary," Int. J. Mech. Sci., vol. 52, pp. 1471-1490, November 2010.

[12] Y. H. Cho, "SPOPS statement of methods," Veh. Syst. Dyn., vol. 53, pp. 329-340, March 2015.

[13] N. Zhou, Q. Lv, Y. Yang, and W. Zhang, "<TPL-PCRUN> Statement of methods," Veh. Syst. Dyn., vol. 53, pp. 380-391, March 2015.

[14] J. P. Massat, E. Balmes, J. P. Bianchi, and G. Van Kalsbeek, "OSCAR statement of methods," Veh. Syst. Dyn., vol. 53, pp. 370-379, March 2015.

[15] J. Ambrósio, J. Pombo, P. Antunes, and M. Pereira, "PantoCat statement of method," Veh. Syst. Dyn., vol. 53, pp. 314-328, March 2015.

[16] A. Collina, S. Bruni, A. Facchinetti, and A. Zuin, "PCaDA statement of methods," Veh. Syst. Dyn., vol. 53, pp. 347-356, March 2015.

[17] F. Vesali, M. A. Rezvani, H. Molatefi, and M. Hecht, "Static formfinding of normal and defective catenaries based on the analytical exact solution of the tensile Euler-Bernoulli beam," P. I. Mech. Eng. F-J. Rai., vol. 233, pp. 691-700, August 2019.

[18] ANSYS® Academic Research Mechanical, Release 18.1, Help System, ANSYS Mechanical APDL Structural Analysis Guide, ANSYS, Inc.

[19] E. Jones, T. Oliphant, P. Peterson, and others, SciPy: Open source scientific tools for Python, 2001. 